\title{
Predicting Fingertip Forces by Imaging Coloration Changes in the Fingernail and Surrounding Skin
}

\author{
Yu Sun*, Member, IEEE, John M. Hollerbach, Fellow, IEEE, and Stephen A. Mascaro, Member, IEEE
}

\begin{abstract}
This paper presents an external camera method for measuring fingertip forces by imaging the fingernail and surrounding skin. A 3-D model of the fingernail surface and skin is obtained with a stereo camera and laser striping system. Subsequent images from a single camera are registered to the 3-D model by adding fiducial markings to the fingernail. Calibration results with a force sensor show that the measurement range depends on the region of the fingernail and skin. A generalized least squares model is developed to predict fingertip force given coloration changes, and results for normal and shear force measurement are presented.
\end{abstract}

Index Terms -Force sensing, haptics, human grasping, imaging, registration.

\section{INTRODUCTION}

I T HAS BEEN shown that coloration changes in the fingernail due to fingertip pressure can serve to transduce fingertip force [14], [15]. Pressure at the fingerpad affects blood flow at the fingernail, which causes a nonuniform pattern of color change in the fingernail area. By measuring the intensity changes at several points of the fingernail, the fingertip force can be deduced after a calibration procedure.

Previously, Mascaro and Asada [14], [15] have proposed a photoplethysmograph (PPD) sensor that has an array of inferred LEDs and photodetectors. It is embedded in a custom-fabricated artificial nail made of epoxy. The artificial nail attaches to the back of the fingernail with an adhesive, and wires are routed out for interface with a computer. The PPD sensor response was linear up to $1 \mathrm{~N}$ normal force, and beyond $1 \mathrm{~N}$, there was a nonlinear leveling off [14]. The sensor predicted normal force to within $1 \mathrm{~N}$ accuracy in the range of $2 \mathrm{~N}$ and shear force to within $0.5 \mathrm{~N}$ accuracy in the range of $3 \mathrm{~N}$. The relation between sensor response and force differs among subjects and different fingernails for the same subject. Therefore, a calibration procedure is required for every fingernail.

In the literature on human grasping [3], [6], [27] instrumented objects are typically created that incorporate miniature six-axis force/torque sensors at predefined grasp points (e.g., [24]-[26]

Manuscript received September 19, 2007; revised March 10, 2008. First published June 10, 2008; current version published September 26, 2008. This work was supported by the National Institute of Health (NIH) under Grant 1R21EB004600-01A2. Asterisk indicates corresponding author.

*Y. Sun was with the School of Computing, University of Utah, Salt Lake City, UT 84112 USA. He is now with Mitsubishi Electric Research Laboratories, Cambridge, MA 02139 USA (e-mail: ysun@cs.utah.edu).

J. M. Hollerbach is with the School of Computing, University of Utah, Salt Lake City, UT 84112 USA (e-mail: jmh@cs.utah.edu).

S. A. Mascaro is with the Department of Mechanical Engineering, University of Utah, Salt Lake City, UT 84112 USA (e-mail: smascaro@mech.utah.edu).

Color versions of one or more of the figures in this paper are available online at http://ieeexplore.ieee.org.

Digital Object Identifier 10.1109/TBME.2008.925691 and [28]). Special-purpose objects have to be fabricated to incorporate force sensors. The points of grasping have to be defined beforehand, which means that a subject is not free to grasp an object in different ways or change the grasp points. So far, the grasp geometries have been limited to cylindrical grasps. If the goal is to characterize grasp forces for a wide variety of everyday objects, where distances and slopes of grasp points vary as well as local surface conditions of curvature, material type, and friction, then a great many special-purpose instrumented objects would have to be made. In addition, miniature six-axis force/torque sensors are expensive.

By contrast, the PPD sensor has the great advantage that objects do not have to be instrumented. Everyday objects can be used, or if objects with special properties such as particular shapes need to be fabricated, it is relatively easy to do so. There is no constraint on how a subject locates or changes grasp points.

Despite the advantages, the PPD sensor has some disadvantages. The sensor needs to be fabricated to fit each fingernail. Other limitations are the sparse sampling of the fingernail and the lack of imaging of the surrounding skin, whose coloration change has been found to transduce fingertip force well also. Besides normal and shear forces, other factors that influence fingernail coloration include shear torque, the contact orientation, the curvature of the contact, and the distal interphalangeal (DIP) joint angle. They all combine to affect the coloration pattern, but a fixed sparse sampling of the fingernail image is not sufficient to separate the influences of these factors.

This paper presents an alternative approach: an external camera system that provides a fuller imaging of the back of the fingertip. The use of an external camera system presents challenges of keeping the fingernail in view, the lighting environment, and registration. None of these challenges is an issue with the PPD sensor, since the sensor is fixed to the back of the nail and the lighting environment is controlled. Nevertheless, the high resolution of the fingernail image and surrounding skin is an offsetting advantage, provided that these challenges can be met. The external camera approach uses the information of the skin as well as the fingernail, since we have found that the surrounding skin transduces much higher level of force than the fingernail does. Furthermore, the external camera approach does not encumber a subject, and there is no need for sensor fabrication and individual fitting. The existence of low-cost cameras and image processing methods readily performed on PCs makes the instrumentation costs of such an approach relatively low.

In this paper, we consider a fixed fingertip pressing against a flat surface mounted on a six-axis force sensor and imaged by a camera system in a controlled lighting environment. We do not yet consider issues of finger tracking or of handling 


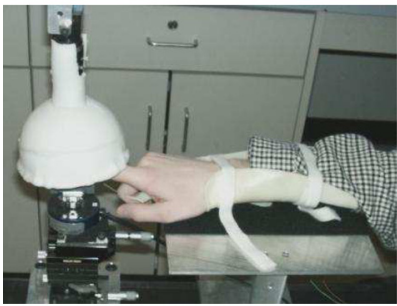

(a)

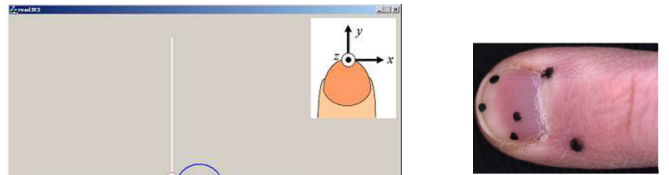

(a)

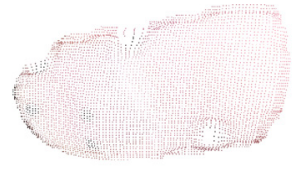

(b) (c)

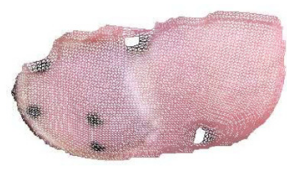

Fig. 2. (a) Fingernail with fiducial marks. (b) The 3-D point cloud from the stereo camera. (c) Triangular 3-D mesh with color mapped from the 2-D image.

(b)

Fig. 1. Experiment setup. (a) Flea 2-D high-resolution camera images a contact plane mounted on a six-axis JR3 force sensor and manual Cartesian stage. A dome light and a molded plastic arm supporter with Velcro strips to provide arm fixation. (b) Visual display feedback.

variable lighting environments in order to explore the fundamental effect of fingertip force versus fingernail coloration, without yet considering such complicating factors. The fingertip orientation is restricted to be flat (nail parallel to the surface). This paper presents the features of the coloration response to fingertip force of the fingernail and surrounding skin. A generalized least squares estimation method is developed to predict fingerpad force from coloration changes.

\section{Calibration Stage}

Fig. 1 shows a calibration stage comprising a six-axis JR3 force sensor (from JR3, Inc.) mounted on a small manual Cartesian stage, a Flea charge-coupled device (CCD) video camera (Point Grey Research, Inc.), and a small lighting dome. A rubber-surface flat plane is mounted on the JR3 force sensor to provide a contact surface; the Cartesian table is adjusted to locate the contact plane beneath a subject's fingertip. The subject's arm is fixated by a molded plastic arm support and Velcro strips; the plastic arm has 2 DOFs for position adjustment. A subject sits in a chair adjustable with 4 DOF for positioning relative to the experimental stage.

The small lighting dome provides a controlled lighting environment so that the images taken at different times are comparable. A reflective hemisphere was created from molded plastic; a hole at the top permits visual access by the Flea camera. LEDs placed along the perimeter reflect off the dome to create uniform lighting on the fingernail surface and to avoid specular reflection.

Images are captured from the Flea camera at $30 \mathrm{fps}$, synchronously with recorded forces from the JR3 force sensor. The force is sampled at $500 \mathrm{~Hz}$. The camera runs at $30 \mathrm{fps}$. They run as two different processes on a computer. In each cycle right before a frame is recorded, the camera handling process requires and stores a current sample of the force from the force-sensorhandling process. We select a Tamron C-mount high-resolution mega pixel lens 23FM16SP so that the Sony ICX204AK CCD chip inside the Flea camera measures an image that is about $8 \mathrm{~cm}$ along the optical axis and is about $4 \times 3 \mathrm{~cm}$ in cross-section. The green channel from the camera's red, green, blue (RGB) color space has been found to produce a larger coloration response and better linearity with force than the other color channels, and is used subsequently.

A visual display [Fig. 1(b)] is provided to guide subjects for calibration. Two of the three dimensions of force read from the JR3 force sensor are represented by position, while the third dimension is represented by the radius of a circle. Colors are used in the actual display. There is a blue circle with a blue cross in the center to represent the actual force applied, as measured by the JR3 force sensor beneath the finger. The $x$-position of the cross represents lateral shear force $f_{x}$, the $y$-position represents longitudinal shear force $f_{y}$, and the size of the circle represents the normal force $f_{z}$. The $x$-position of a white-filled red-edge sphere represents the desired shear force $f_{x}$ and the $y$-position represents the desired shear force $f_{y}$. The circle size of the red circle, whose center follows the cross, represents the desired normal force $f_{z}$.

\section{IMAGE REGISTRATION}

Fingernail locations will vary depending on the grasp and the relative locations of the camera. As a particular fingernail is imaged, it will be necessary to correspond points in the image to a reference image so that calibration results can be applied. In experiments, subjects can translate their fingers and rotate in and out of the contact plane. When the optical axis is not perpendicular to the fingernail and the viewing distance is short, it is necessary to include the curvature of the fingernail.

The reference image needs to be a 3-D surface model fitted to the fingernail. After comparing different surface representations including polygonal meshes, B-spline surfaces, and quadric surfaces, we chose a dense triangle mesh model since it is easiest to adapt to fingernail geometry. 3-D points that form the vertices of triangular meshes are obtained with a Bumblebee BBHICOL-60 (Point Grey Research, Inc.) stereo camera. Since the fingernail is smooth and relatively featureless, it is difficult for the stereo camera system to find corresponding points in the two images. A common computer vision method for such situations is structured light onto the surface, which is easy for stereo vision to match [10]. We employ a Steminc SMM96355SQR laser module to create a $4 \times 4$ grid pattern. A 3 -D data cloud obtained from the stereo camera is shown in Fig. 2(b).

We do not employ the Bumblebee stereo camera for the coloration measurements because its resolution is too low. However, its output is adequate for determining a 3-D mesh model.

To map the high resolution Flea 2-D images to a 3-D model, we employ a well-known technique from computer vision [7] of adding fiducial marks to the fingernail and surrounding skin with a black marker [Fig. 2(a)]. Without fiducial marks, automatic 
feature detection can only find landmarks on the edge of the fingernail and in the surrounding skin since the fingernail is smooth and relatively featureless.

It is necessary that the relative locations of the fiducial markings in the 3-D model be known; this knowledge is obtained using the stereo camera. The fiducial marks are then automatically detected in the 2-D image from the Flea camera [29], [33] and used to compute the extrinsic parameter matrix $[\mathbf{R} \mathbf{t}]$, where $\mathbf{R}$ and $\mathbf{t}$ are the rotation and displacement from the 2-D image to the coordinates of the 3-D model.

The homogeneous coordinates of a point $i$ in the 2-D image $\mathbf{p}_{i}$ and in the 3-D model $\mathbf{P}_{i}$ are

$$
\mathbf{p}_{i}=\left[\begin{array}{lll}
u_{i} & v_{i} & 1
\end{array}\right]^{T}, \quad \mathbf{P}_{i}=\left[\begin{array}{llll}
X & Y & Z & 1
\end{array}\right]^{T}
$$

where the 2-D camera coordinates are $\left(u_{i}, v_{i}\right)$. Let $\mathbf{K}$ be the intrinsic parameter matrix for the camera, and define the $3 \times 4$ transformation

$$
\mathbf{M}=\mathbf{K}\left[\begin{array}{ll}
\mathbf{R} & \mathbf{t}
\end{array}\right]=\left[\begin{array}{lll}
\mathbf{m}_{1} & \mathbf{m}_{2} & \mathbf{m}_{3}
\end{array}\right]^{T} .
$$

The transform relation between the two coordinates is $\mathbf{p}_{i}=$ $\mathbf{M P}_{i}$. Hence,

$$
\begin{aligned}
& \mathbf{m}_{1}^{T} \mathbf{P}_{i}-\left(\mathbf{m}_{3}^{T} \mathbf{P}_{i}\right) u_{i}=0 \\
& \mathbf{m}_{2}^{T} \mathbf{P}_{i}-\left(\mathbf{m}_{3}^{T} \mathbf{P}_{i}\right) v_{i}=0 .
\end{aligned}
$$

With six fiducial marks, the parameters in $\mathbf{M}$ can be calibrated with linear least squares. A registration result is shown in Fig. 2(c).

In practice, if the axis of the 2-D camera is always located perpendicular to the fingernail, the curvature of the fingernail can be neglected. A much simpler and faster 2-D to 2-D registration would be sufficient. Natural landmarks on the edges of the fingernail and surrounding skin can be automatically detected for 2-D homography mapping. A detailed 2-D to 2-D registration is described in [32].

\section{COLORATION RESPONSE}

After registration, the color changes in the fingernail and surrounding skin corresponding to the force on the fingertip are studied point by point. For all subjects, we have found that different regions in the fingernail and surrounding skin responds differently to the force.

For simplicity of mathematical formulation, we only consider 1-D force in this section. The derived conclusions hold for the 3-D force situation.

\section{A. Response Model}

After investigating the response curves of all points in the fingernails and surrounding skin of all subjects, we found that the shape of the response curves was sigmoidal. Fig. 3(a) shows the response of a typical point in the fingernail (open circles). The color barely changes for small forces until $2 \mathrm{~N}$, and then, starts to change significantly until the force reaches $4.5 \mathrm{~N}$. A generalized sigmoid function [34] is fit to the data

$$
h\left(x ; a, b, c, x_{0}\right)=a+\frac{b}{1+e^{c\left(x-x_{0}\right)}}
$$

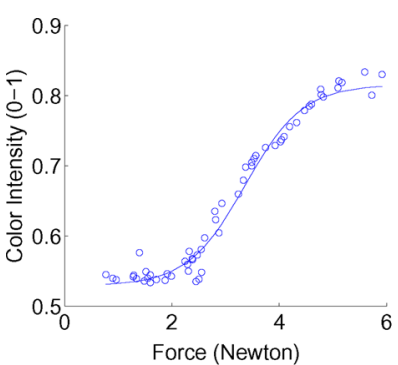

(a)

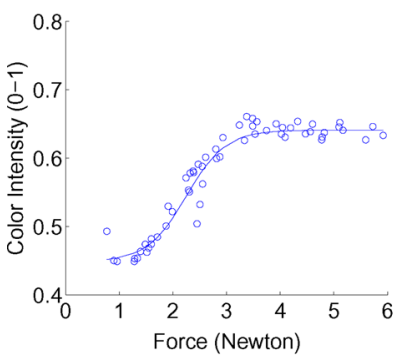

(c)

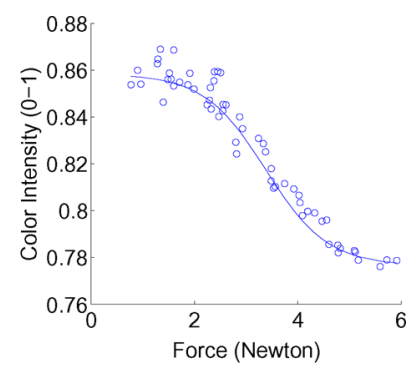

(b)

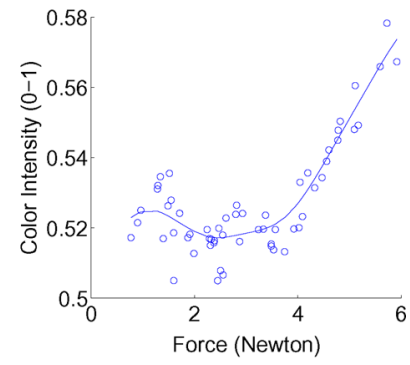

(d)
Fig. 3. Coloration changes at different location. (a) Measured color response (circles) versus modeled sigmoidal response (solid line) of a typical point in the fingernail to force applied on the fingertip. (b) Color intensity changes from bright to dark with an increasing force. (c) Point starts to respond at around $1 \mathrm{~N}$ and saturates after around $3 \mathrm{~N}$. (d) Point starts to respond at around $4 \mathrm{~N}$ and continues to increase.

where $a, b, c$, and $x_{0}$ are the regression parameters. The modeled response is shown as a line in Fig. 3(a). The parameters have geometric meaning. $a$ is the lower asymptote, $b$ is the response range, $x_{0}$ is the force of maximum response rate, and $c$ is the relative response rate. Other examples at different points in the fingernail are shown in Fig. 3(b)-(d).

The color changes in the fingernail and surrounding skin reflect the blood distribution in the fingernail. When there is pressure on the fingertip, the blood vessels deform. The volume of the blood vessel was found to respond sigmoidally to force in [13]. Blood is squeezed out from some vessels due to compression, while pooled in other vessels due to tension [16]. In compression regions, the response looks like Fig. 3(a) since the area is whitening with blood loss. In tension regions, the blood is pooling and so the coloration response darkens as in Fig. 3(b).

Different regions start to respond and saturate at different force levels. The example point in Fig. 3(c) starts to respond at $1 \mathrm{~N}$ and saturates at $3 \mathrm{~N}$. The example point in Fig. 3(d) starts to respond at a relatively high force level of $4 \mathrm{~N}$, and continues to increase even at the high end of our interest range.

\section{B. Segment Selection}

Using $Q-Q$ plot [9], the residual errors of $h_{i}$ of a point $i$ in the fingernail and surrounding skin on a force $f$ are found to satisfy a normal distribution [23]. Therefore, the response is modeled as a conditional Gaussian

$$
p\left(h_{i} \mid f\right)=\frac{1}{K_{1}} e^{-\left(h_{i}-\bar{h}_{i}\right)^{2} / 2 \sigma_{i}^{2}}
$$


where $\bar{h}_{i}$ and $\sigma_{i}$ are the mean and the variance, respectively, and $K_{1}$ is a constant. For all points from 1 to $m$, the joint distribution is

$$
p(\mathbf{h} \mid f)=\frac{1}{K_{1}} e^{-(1 / 2)(\mathbf{h}-\overline{\mathbf{h}})^{T} \Sigma^{-1}(\mathbf{h}-\overline{\mathbf{h}})}
$$

where $\mathbf{h}=\left[h_{1} \cdots h_{m}\right]^{T}$ and $\Sigma$ is the covariance of $\mathbf{h}$.

The distribution of a force on the condition of measured color of all the points is $p(f \mid \mathbf{h})$. With Bayes rule

$$
p(f \mid \mathbf{h})=\frac{p(\mathbf{h} \mid f) p(f)}{p(\mathbf{h})} .
$$

If we assume that the force distribution is uniform, $p(f)$ is a constant. Also, $p(\mathbf{h})$ is independent of $f$. Therefore, we write

$$
p(f \mid \mathbf{h})=\frac{1}{K_{2}} e^{-(1 / 2)(\mathbf{h}-\overline{\mathbf{h}})^{T} \boldsymbol{\Sigma}^{-1}(\mathbf{h}-\overline{\mathbf{h}})}
$$

where the constant $K_{2}=K_{1} p(\mathbf{h}) / p(f)$.

For a small segment, the relation between $f$ and $h_{i}$ is piecewise linear

$$
h_{i}(f)=\alpha_{i}(f)+\beta_{i}(f) f+\varepsilon_{i}
$$

where $\alpha_{i}(f)$ and $\beta_{i}(f)$ are piecewise linear parameters, and from observation, we can assume that $\varepsilon_{i}$ does not change with $f$ and is Gaussian. Since $E\left[\varepsilon_{i}\right]=0$, then

$$
\bar{h}_{i}(f)=\alpha_{i}(f)+\beta_{i}(f) f .
$$

Thus,

$$
\begin{aligned}
& p(f \mid \mathbf{h}) \\
& \quad=\frac{1}{K} e^{-(1 / 2)(\mathbf{h}-\boldsymbol{\alpha}(f)-\boldsymbol{\beta}(f) f)^{T} \boldsymbol{\Sigma}^{-1}(\mathbf{h}-\boldsymbol{\alpha}(f)-\boldsymbol{\beta}(f) f)}
\end{aligned}
$$

where $\quad \boldsymbol{\alpha}(f)=\left[\alpha_{1}(f) \ldots \alpha_{m}(f)\right]^{T}$ and $\boldsymbol{\beta}(f)=\left[\beta_{1}(f) \ldots\right.$ $\left.\beta_{m}(f)\right]^{T}$.

The distribution is determined by the quadratic form

$$
\Delta^{2}=(\mathbf{h}-\boldsymbol{\alpha}(f)-\boldsymbol{\beta}(f) f)^{T} \boldsymbol{\Sigma}^{-1}(\mathbf{h}-\boldsymbol{\alpha}(f)-\boldsymbol{\beta}(f) f) .
$$

Expressing in terms of $f$

$$
\Delta^{2}=\left(f-\frac{\mathbf{h}-\boldsymbol{\alpha}(f)}{\boldsymbol{\beta}(f)}\right)^{T} \boldsymbol{\beta}(f)^{T} \boldsymbol{\Sigma}^{-1} \boldsymbol{\beta}(f)\left(f-\frac{\mathbf{h}-\boldsymbol{\alpha}(f)}{\boldsymbol{\beta}(f)}\right) .
$$

From (10), the variance of the distribution $p(f \mid \mathbf{h})$ is

$$
\sigma_{f \mid \mathbf{h}}^{2}=\left(\boldsymbol{\beta}(f)^{T} \boldsymbol{\Sigma}^{-1} \boldsymbol{\beta}(f)\right)^{-1} .
$$

For simplicity, we assume that the colors of all the points are independent. Hence, the off-diagonal elements of $\Sigma=$ $\operatorname{diag}\left(\sigma_{1}^{2} \ldots \sigma_{m}^{2}\right)$ are zeros, and

$$
\sigma_{f \mid \mathbf{h}}^{2}=\frac{1}{\frac{\beta_{1}(f)^{2}}{\sigma_{1}^{2}}+\cdots+\frac{\beta_{m}(f)^{2}}{\sigma_{m}^{2}}} .
$$

On the condition of the colors measured from a set of points in the fingernail and surrounding skin, the distribution of the force has the variance as formulated in (12). The variance of the force depends on the variances of the colors and the slopes of the color responses at that force level. Each color measurement of a point $i$ decreases the variance of $f$ by $\beta_{i}(f)^{2} / \sigma_{i}^{2}$. When the

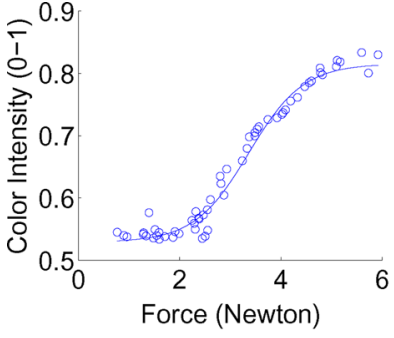

(a)

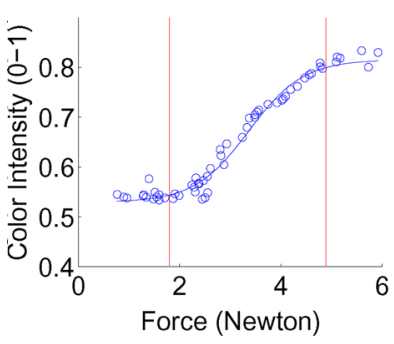

(c)

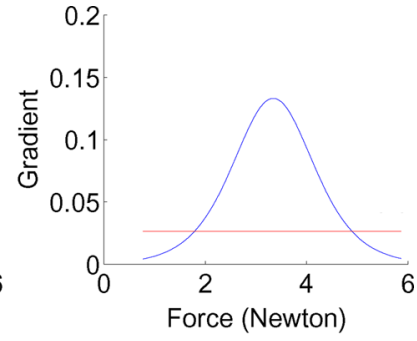

(b)

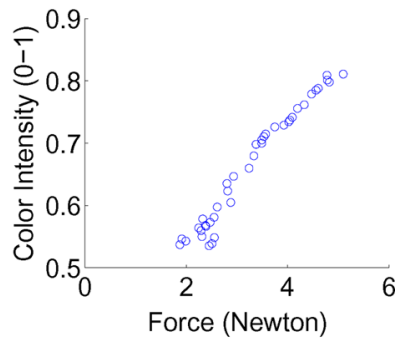

(d)
Fig. 4. Selection of correlation range. (a) Sigmoid fit of the color response. (b) Gradients of the sigmoid function. The one-fifth of the maximum gradient is indicated. (c) and (d) Selected segment.

slope $\beta_{i}(f)$ is large and the variance of the color $\sigma_{i}$ is small, its contribution to decrease the variance of the force is substantial. On the other hand, when the slope $\beta_{i}(f)$ is small and the variance of the color $\sigma_{i}$ is large, its contribution is negligible.

After sigmoid regression fitting, the gradient of the response curve [Fig. 4(a)] is computed [Fig. 4(b)]. Segments of the sigmoid with gradients lower than one-fifth of the maximum gradient of the sigmoid curve are not used. Segments with large gradients [e.g., Fig. 4(c) and (d)] are selected for force estimation. The force range of the selected segment is called the transducing range. The color intensity range of the selected segment is called the response range. For this example point, the transducing range is $1.8-4.9 \mathrm{~N}$ and the response range is $0.54-0.80 \mathrm{~N}$.

Different points in the fingernail and surrounding skin have different transducing ranges. Fig. 5 shows the start point color map (top two rows) and the saturation point color map (bottom two rows) of one subject. The dark points in each figure are the regions of the fingernail and surrounding skin with the associated force levels.

1) Most points in the front of the fingernail start to respond at a force level of 2-3 $\mathrm{N}$ and saturate at 5-6 $\mathrm{N}$.

2) Most areas in the middle of the fingernail start to respond at $0-1 \mathrm{~N}$. Some of these areas saturate at $1-2 \mathrm{~N}$, while others saturate at 2-3 N.

3) Some areas on the skin surrounding the fingernail start to respond at 3-4 $\mathrm{N}$ and some start to respond at $4-5 \mathrm{~N}$. They all saturate at a force larger than $6 \mathrm{~N}$.

By combining all areas, the fingernail coloration can transduce forces from 0 to $10 \mathrm{~N}$ for this subject. Different subjects have different response maps; individual calibration is required.

In practice, since fitting a sigmoid function is timeconsuming, the responses are fitted with locally weighted least squares [17]. As shown in Fig. 6, the gradient of the response 


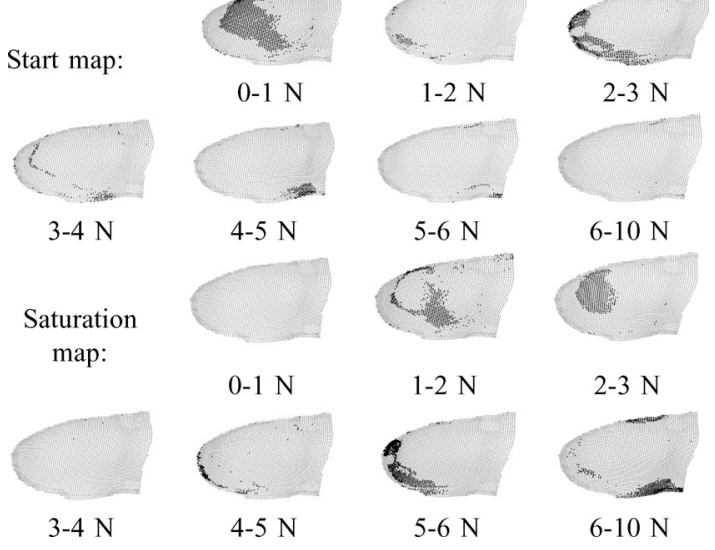

Fig. 5. Regions of the finger and their force starting and saturation levels.

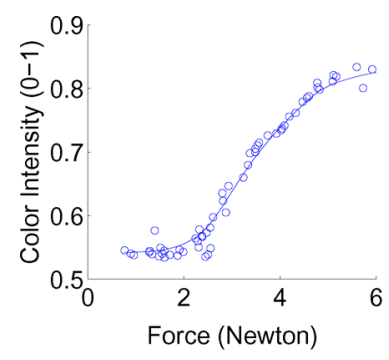

(a)

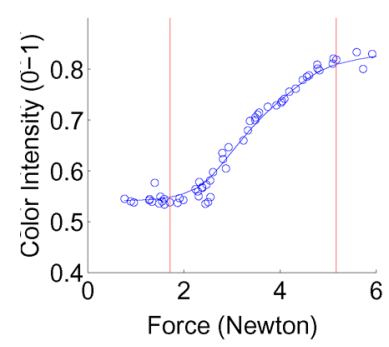

(c)

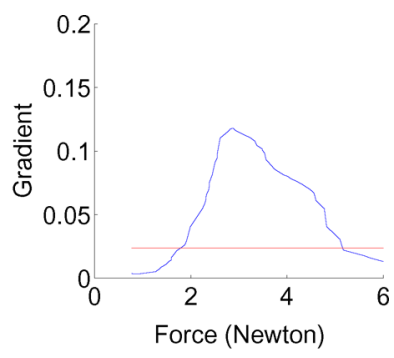

(b)

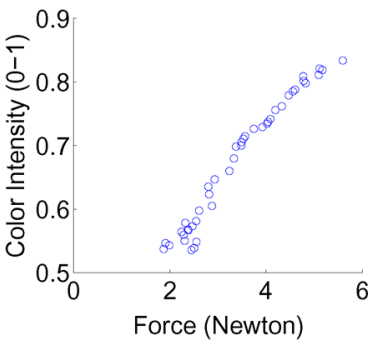

(d)
Fig. 6. Segment selection with local-weighted least square.

curve is computed locally, and the segmentation selection is carried out in the same way as for the sigmoidal curve. The locally weighted least squares fitting is carried as follows.

1) Locally weighted linear regression is used to fit the response curve [17]. The weighting function is $w_{k}=$ $e^{-D\left(f_{k}, f_{i}\right)^{2} / K_{w}^{2}}$, where $i$ is the index of the query point, $k$ is the index of points around $i, K_{w}$ is the kernel size, $D$ is a constant, and $\left(f_{k}, f_{i}\right)^{2}$ refers to the distance from a point $i$ to the query point $k$. Here, it is the distance along the force axis. It gives large weight to the points close to the query point and small weights to distant points. This curve fitting emphasizes local information, which can pick up turning points. A typical result is shown in Fig. 6(a).

2) Local gradients on the fitted curve are calculated by differentials [Fig. 6(b)].

3) The gradient threshold $T_{\mathrm{th}}$ is one-fifth of the maximum gradient of the curve. The segment with gradient larger than $T_{\text {th }}$ is selected [Fig. 6(c) and (d)].

\section{LINEAR RESPONSE REGIONS}

As shown in Fig. 4(d), with segment selection, the selected middle segment of the sigmoid curve is very close to linear. Due to the simplicity of a linear model, it would be attractive to model the response with linear models.

With experiments, we found that certain areas of the fingernail and surrounding skin show a good linear response of coloration to fingertip force, others do not. Within the response range of mesh element $i$, a linear model relating coloration intensity $h_{i j}$ to a force component $f_{j}$ for $j=1, \ldots, n$ reading pairs is fit using ordinary least squares

$$
h_{i j}=a_{i 0}+a_{i 1} f_{j}, \quad j=1, \ldots, n
$$

where $a_{i 0}$ and $a_{i 1}$ are the linear fitting parameters for mesh element $i$. The goodness of fit is determined by thresholding the correlation coefficient $r_{i}$ [5]

$$
r_{i}=\frac{\sum_{j=1}^{n}\left(f_{j}-\bar{f}\right)\left(h_{i j}-\bar{h}_{i}\right)}{\sqrt{\sum_{j=1}^{n}\left(f_{j}-\bar{f}\right)^{2} \sum_{j=1}^{n}\left(h_{i j}-\bar{h}_{i}\right)^{2}}}
$$

where $\bar{f}$ and $\bar{h}_{i}$ are the averages of the force and coloration readings, respectively. A threshold of $r_{i}=0.8$ is chosen to exclude those mesh elements whose response is not linear enough to be employed for force prediction.

\section{Generalized Least SQuares Modeling}

Using the good mesh elements with coloration readings $h_{i}$, a generalized least squares estimator [19] is applied to predict fingertip force vector $\mathbf{f}=\left(f_{x}, f_{y}, f_{z}\right)$ for a single reading. Generalize the model (13) to predict multiple force components

$$
h_{i}=a_{i}+\mathbf{b}_{i}^{T} \mathbf{f}+\varepsilon_{i}
$$

where $a_{i}$ and $\mathbf{b}_{i}=\left(b_{1}, b_{2}, b_{3}\right)$ are unknown parameters, and $\varepsilon_{i}$ is the residual error. The parameters $a_{i}$ and $\mathbf{b}_{i}$ are fitted in their response ranges using ordinary least squares similar to the one-component force model (13). Combine all readings $h_{i}$ into a vector $\mathbf{h}$, the parameters $a_{i}$ into a vector $\mathbf{a}$, the parameters $\mathbf{b}_{i}$ into the rows of a matrix $\mathrm{B}$, and the errors into a vector $\epsilon$. Then, stack (15) results

$$
\mathbf{h}-\mathbf{a}=\mathbf{B} \mathbf{f}+\epsilon .
$$

The response model (5) can be written as

$$
p(\mathbf{h} \mid \mathbf{f})=\frac{1}{K} e^{-(1 / 2)(\mathbf{h}-\overline{\mathbf{h}})^{T} \Sigma^{-1}(\mathbf{h}-\overline{\mathbf{h}})}
$$

where $\overline{\mathbf{h}}$ is the average of $\mathbf{h}$ and $K$ is a constant. The covariance matrix $\Sigma$ is estimated from the data. The generalized least squares estimate of the force is

$$
\hat{\mathbf{f}}=\left(\mathbf{B}^{T} \boldsymbol{\Sigma}^{-1} \mathbf{B}\right)^{-1} \mathbf{B}^{T} \boldsymbol{\Sigma}^{-1}(\mathbf{h}-\mathbf{a}) .
$$

The mesh elements are weighted by the uncertainties $\Sigma^{-1}$ to produce the best force estimates.

When the prior force probabilities are not uniformly distributed, it would make the Bayesian estimator different from least squares. However, the prior p.d.f. $p(f)$ can be meaningfully 


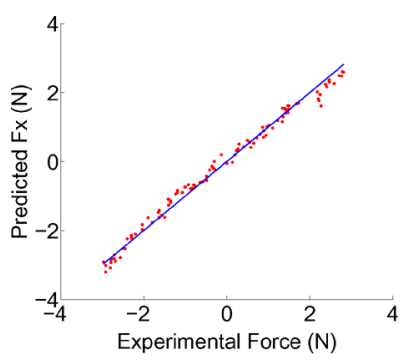

(a)

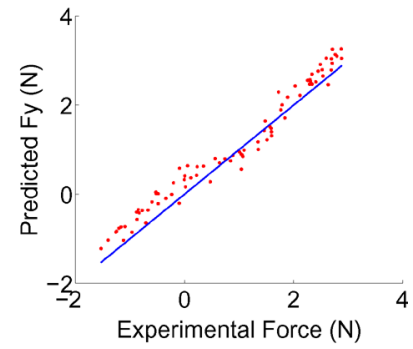

(b)

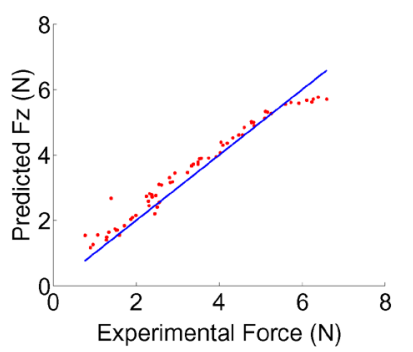

(c)

Fig. 7. Predicted responses for subject 1 exerting forces primarily in the (a) $f_{x}$, (b) $f_{y}$, and (c) $f_{z}$ directions.

chosen in general because the information conveyed by the measurements far outweighs that contained in the prior p.d.f. and the final parameter estimate is not very sensitive to the prior [19].

\section{CAlibration AND VERIFICATION}

To verify the system, a first set of experiments was carried out to determine how well each force component could be determined in isolation. Seven subjects participated. Three of them are east Asians; one of them is Asian Indian and has dark skin; three of them are white. Three of them are females and four of them are males.

Subjects used their index fingers to press on the rubber plate mounted on the JR3 force sensor while the camera monitored the coloration change of the index finger. Subjects were asked to produce normal force $f_{z}$ or shear force $f_{x}$ or $f_{y}$ under the guidance of the visual display. For each direction of force, three sets of data were taken. The first two sets were used for calibration and the third set was used for verification. The estimation equation (18) was simplified to predict only one component of force.

Fig. 7 shows examples of separate predictions of shear force ( $f=f_{x}$ or $\left.f=f_{y}\right)$ and normal force $\left(f=f_{z}\right.$ ) for slow force ramps for one subject. Table I shows the rms error of the prediction results for this subject (subject 1) plus six other subjects. The predictions in all directions show good linearity. The normal force for this subject saturates above $6 \mathrm{~N}$, which is a typical result. Some subjects have saturation levels as low as $4 \mathrm{~N}$, while others have saturation levels above $8 \mathrm{~N}$. The saturation level limits the magnitude of grasp forces that can be measured by the coloration effect. The shear force levels are less because contact is broken. For subject 1's $6 \mathrm{~N}$ force range, the inaccuracy of prediction ranges from $2.5 \%\left(-f_{x}\right)$ to $7.8 \%\left(-f_{y}\right)$. We also computed the multidimensional coefficients of deter-
TABLE I

rms Errors (N) of Prediction for Force Components for Seven Subjects

\begin{tabular}{|l|ccccc|}
\hline Subject & $+f_{x}$ & $-f_{x}$ & $+f_{y}$ & $-f_{y}$ & $f_{z}$ \\
\hline 1 & 0.17 & 0.15 & 0.27 & 0.47 & 0.28 \\
\hline 2 & 0.35 & 0.36 & 0.42 & 0.44 & 0.27 \\
\hline 3 & 0.52 & 0.52 & 0.11 & 0.35 & 0.27 \\
\hline 4 & 0.49 & 0.49 & 0.32 & 0.34 & 0.30 \\
\hline 5 & 0.60 & 0.60 & 0.44 & 0.51 & 0.34 \\
\hline 6 & 0.11 & 0.11 & 0.38 & 0.31 & 0.33 \\
\hline 7 & 0.29 & 0.28 & 0.38 & 0.30 & 0.34 \\
\hline
\end{tabular}
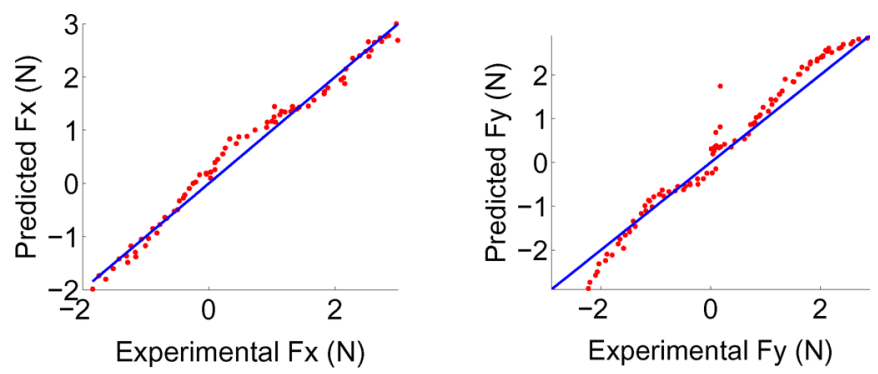

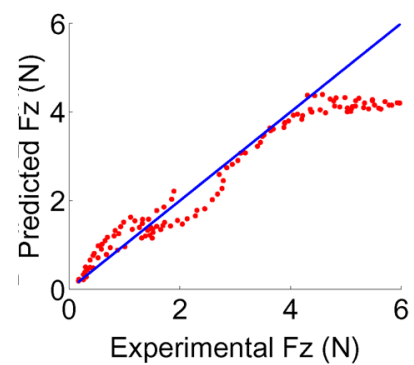

(a)

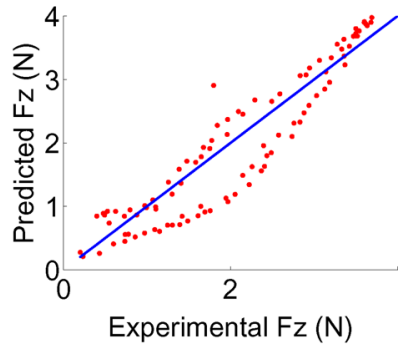

(b)
Fig. 8. Simultaneous prediction of normal $f_{z}$. (a) With shear force $f_{x}$. (b) With shear force $f_{y}$.

mination [23], which were above 0.9 and often above 0.95 . This shows that averaging the responses of the lower correlation, individual mesh elements have produced the desired effect of increased accuracy.

The second set of experiments was conducted to determine whether a shear force component (either $f_{x}$ or $f_{y}$ ) could be predicted simultaneously with normal force $f_{z}$. Again, (18) was simplified to predict only two force components. Subjects were requested to exert a shear force primarily in the $f_{x}$ - or in the $f_{y}$-direction. The subjects had to generate some normal force $f_{z}$ as well to maintain frictional contact. A calibration model is developed from one set of data, then used to predict another dataset. The results are shown in Fig. 8 for one subject; responses for other subjects are similar. For the $x$-direction, the prediction errors are $0.17 \mathrm{~N}$ in $f_{x}$ and $0.30 \mathrm{~N}$ in $f_{z}$ [Fig. 8(a)]. For the $y$-direction, the prediction errors are $0.27 \mathrm{~N}$ in $f_{y}$ and $0.48 \mathrm{~N}$ in $f_{z}$ [Fig. 8(b)].

\section{TIME COURSE OF COLORATION EFFECT}

The viscoelasticity of the fingertip pulp and circulation dynamics will affect how fast the fingernail coloration changes in response to fingertip force changes. The mechanical properties of the fingertip were modeled by Pawluk and Howe [20], [21] 

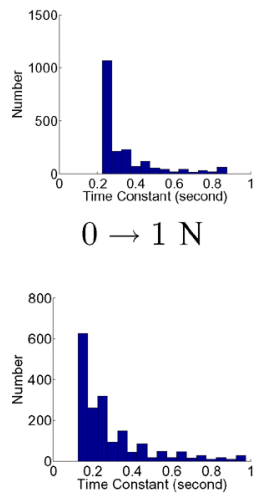

$1 \rightarrow 0 \mathrm{~N}$
$0 \rightarrow 1 \mathrm{~N}$

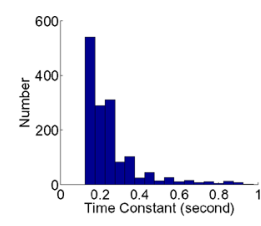

$1 \rightarrow 2 \mathrm{~N}$

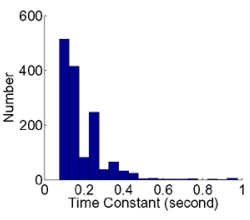

$2 \rightarrow 1 \mathrm{~N}$

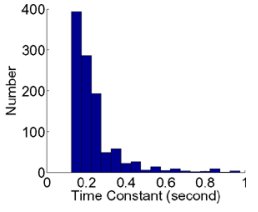

$2 \rightarrow 3 \mathrm{~N}$

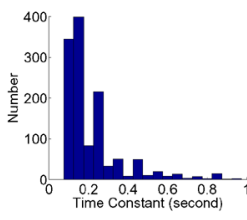

$3 \rightarrow 2 \mathrm{~N}$
Fig. 9. Response histograms for $1 \mathrm{~N}$ steps for both loading and unloading from 0 to $3 \mathrm{~N}$.

with a viscoelastic model with three time constants, whose values were determined to be $0.004,0.07$, and $1.4 \mathrm{~s}$. Over $75 \%$ of the magnitude of the response was due to the first two fast terms whose time constants are less than $0.1 \mathrm{~s}$. From pulsatile pressure variation in the data, the blood flow is already restored by the time that the third term dominates. Experimental evidence from Mascaro and Asada [14] verified that the time constant of the response of the blood flow is between 0.1 and $0.4 \mathrm{~s}$, depending on which part of the fingernail is observed.

Using the more recent methods described before to identify good mesh elements, we have verified that the coloration effect is reasonably fast. A series of force steps was applied by a linear motor to the fixated finger. For each force step, the first-order time constant [22] was calculated for each mesh element. We found that the time constants tend to cluster around $0.2 \mathrm{~s}$, and the loading and unloading responses in the same range are relatively similar. A few mesh elements have responses much slower than $0.2 \mathrm{~s}$. Fig. 9 shows the histograms of the time constants for $1 \mathrm{~N}$ force steps from 1 to $3 \mathrm{~N}$ for both loading and unloading of one subject. More results are shown in our previous work [31].

Given time constants around 0.2 or $0.3 \mathrm{~s}$, the rate of fingertip force change would have to be kept relatively slow to employ the coloration effect. For fast force prediction, since the time constants are fairly consistent and calibratable, time compensation can be applied. An experiment was carried out to test how the dynamic features of the fingernail coloration affect the model and the possibility of time compensation. The training is carried out with slow force and the model is tested on a fast dataset. Fig. 10(a) shows the prediction result without time compensation. The shapes of the actual versus predicted force are fairly similar, but they are displaced in time. The time compensation result is shown in Fig. 10(b), which is fairly good.

\section{DISCUSSION}

This paper has shown that by imaging, the coloration changes in the fingernail and surrounding skin with an external camera, normal and shear forces can be estimated with an accuracy of $5 \%-10 \%$ for a force range of up to $10 \mathrm{~N}$. Individual calibration is required, and a weighted least squares estimator was proposed.

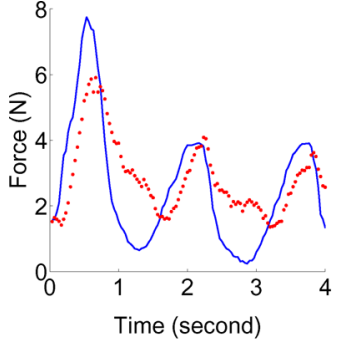

(a) (b)

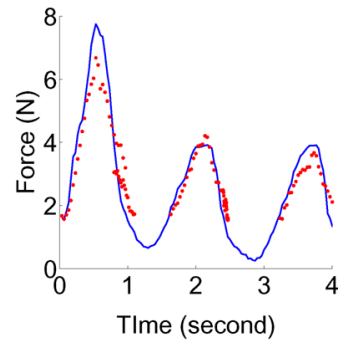

Fig. 10. Force prediction temporal compensation. (a) Prediction result for fast forces, without considering the dynamic features. (b) Prediction result for fast force with linear phase shifting.

The dense imaging of the fingernail, and the finding that the surrounding skin transduces the higher force levels, validate the external camera approach as opposed to the limited sampling of the earlier PPD sensor [14].

Our results show a complex pattern of coloration versus force across the fingernail and skin. Generally speaking, the middle region of the fingernail has a low force range $(0-2 \mathrm{~N})$, the front region has an intermediate force range $(2-6 \mathrm{~N})$, and the surrounding skin has a high force range $(3$ to $>6 \mathrm{~N})$. The saturation level varies with subject: sometimes less than $6 \mathrm{~N}$, sometimes up to $10 \mathrm{~N}$. To estimate the fingertip force over the entire range from $0 \mathrm{~N}$ to saturation requires combining readings from all fingernail and skin regions.

This complex pattern explains the saturation level of $2 \mathrm{~N}$ and accuracy of $0.5 \mathrm{~N}$ in a range of $0-1 \mathrm{~N}$ for the PPD sensor [14]. A few points on the nail were imaged, typically in the middle. The fixed sampling location of half a dozen points meant that the best responding regions could not be identified and combined. The generalized least square estimator may also yield greater accuracies than the basic least squares estimator in [14].

Better accuracies might be possible by designing an automated calibration system. In this paper, subjects themselves generated fingertip forces under the guidance of a graphical display. Apart from the issue of efficiency and time taken to obtain a good calibration, self-generated shear forces are typically linear with normal force, and hence, are not independently produced. This reflects the well-known relation between grip force and load force in grasping [35]. A calibration procedure would ideally vary the shear/normal force ratio while maintaining frictional contact. A motorized calibration stage might lead to a more robust and accurate estimation.

The dynamic features presented here show that for the same measuring point, the time constants are different for different force levels and directions (loading and unloading), and the typical time constant is around $0.2 \mathrm{~s}$. The system's response speed is limited by the speed of artery deformation and blood flow. However, for many grasp studies and applications, the finger force frequency is fairly low. For example, during surgical training, a majority of the frequency content is below $5 \mathrm{~Hz}$; during tissue grasps, the average grasp force frequency is approximately $1 \mathrm{~Hz}[8]$. 
Although not as accurate as employing an external force sensor at predefined grasp points, our method has compensating advantages. Everyday objects can be employed, and grasp points do not have to be predefined. The fingerpad naturally comes into contact with the object surface, rather than having surfaces defined by an external force sensor. The use of external cameras is a low-cost sensing means.

The usable force range, accuracy, and response speed from our imaging system correspond perfectly to typical fingertip forces during contact. Pawluk and Howe [20] reported that forces between 0 and $2 \mathrm{~N}$ are the most relevant for grasping and typing. Jones [12] has found that a human is capable of controlling a constant finger force in the range of 2-6 $\mathrm{N}$ with average error of $6 \%$ with visual feedback and natural haptic sense. Also, Mascaro and Asada [14] found that the force that a human subject can comfortably apply for an extended time is about $3 \mathrm{~N}$. For precision grasps, such as surgeon grasps, the majority of the frequency content is below $5 \mathrm{~Hz}$ [8].

The individual calibration is currently a drawback. Our study [32] has shown that after shape normalization, all subjects studied have the same responding regions for different directions of force. The consistency across subjects may ease the individual calibration process. In the future, we will investigate how to use the consistent response in calibration.

This research investigated the accuracy of fingertip force prediction based on coloration changes for relatively ideal conditions: the fingerpad contacted a flat surface in a parallel orientation, the finger was kept stationary and in easy view of a camera, and a controlled lighting environment was used. The rationale for these restrictions is in part to ascertain the fundamental limitations of the coloration effect, without yet bringing in technological issues of how to track the fingernail with a camera system or how to handle varied lighting environments. For fingernail viewing, it is possible to place miniature portable cameras and lighting domes on an exoskeleton worn by the user. If the grasping workspace does not vary too much, a number of cameras can be arranged around the hand. New computer vision techniques are being developed that handle varied lighting environments, and are particularly good at eliminating the influence of specular reflection [18].

Future work will also investigate different contact conditions. The curvature of contact will be varied, such as a point indenter or a line indenter. The orientation of the fingerpad on the contacted surface will be varied. Adding more variables will certainly be a challenge for the proposed method. Once again, critical to these studies will be the development of an automated calibration system.

\section{ACKNOWLEDGMENT}

Portions of this research have been published in [30] and [31].

\section{REFERENCES}

[1] A. Avolio, "The finger volume pulse and assessment of arterial properties," J. Hypertens., vol. 20, pp. 2341-2343, 2002.

[2] G. Baud Bovy and J. F. Soechting, "Two virtual fingers in the control of the tripod grasp," J. Neurophysiol., vol. 86, pp. 604-615, 2001.
[3] G. Baud-Bovy and J. F. Soechting, "Factors influencing variability in load forces in a tripod grasp," Exp. Brain Res., vol. 143, pp. 57-66, 2002.

[4] A. Benali, P. Richard, and P. Bidaud, "Design, control and evaluation of a six DOF force feedback interface for virtual reality applications," in Proc. IEEE Int. Workshop Robot Hum. Interact. (ROMAN'99), 1999, pp. 338-343.

[5] J. S. Bendat and A. G. Piersol, Engineering Applications of Correlation and Spectral Analysis. New York: Wiley, 1980.

[6] A. Bicchi and V. Kumar, "Robotic grasping and contact: A review," in Proc. IEEE Int. Conf. Robot. Automat., San Francisco, CA, 2000, vol. 1, pp. 348-353.

[7] A. G. Brown, "A survey of image registration techniques," ACM Comput. Surveys, vol. 24, pp. 226-276, 1992.

[8] J. D. Brown, J. Rosen, L. Chang, M. Sinanan, and B. Hannaford, "Quantifying surgeon grasping mechanics in laparoscopy using the blue DRAGON system," in Studies in Health Technology and InformaticsMedicine Meets Virtual Reality, vol. 98, Amsterdam, The Netherlands: IOS Press, Jan. 2004, pp. 34-36.

[9] J. M. Chambers, W. S. Cleveland, B. Kleiner, and P. A. Turkey, Graphical Methods for Data Analysis.. $\quad$ Boca Raton, FL: Chapman \& Hall/CRC, 1983.

[10] D. Forsyth and J. Ponce, Computer Vision-A Modern Approach. Englewood Cliffs, NJ: Prentice-Hall, 2003.

[11] A. P. Georgopoulos, A. B. Schwartz, and R. E. Kettner, "Neuronal population coding of movement direction," Science, vol. 233, pp. 1416-1419, 1986.

[12] L. A. Jones, "Perception and control of finger forces," in Proc. ASME Dyn. Syst. Control Div., 1998, vol. 64, pp. 133-137.

[13] G. J. Langewouters, A. Zwart, R. Busse, and K. H. Wesseling, "Pressurediameter relationships of segments of human finger arteries," Clin. Phys. Physiol. Meas., vol. 7, pp. 43-55, 1986.

[14] S. A. Mascaro and H. H. Asada, "Photoplethysmograph fingernail sensors for measuring finger forces without haptic obstruction," IEEE Trans. Robot. Autom., vol. 17, no. 5, pp. 698-708, Oct. 2001.

[15] S. A. Mascaro and H. H. Asada, "Measurement of finger posture and three-axis fingertip touch force using fingernail sensors," IEEE Trans. Robot. Autom., vol. 20, no. 1, pp. 26-35, Feb. 2004.

[16] S. A. Mascaro and H. H. Asada, "The common patterns of blood perfusion in the fingernail bed subject to fingertip touch force and finger posture,' Haptics-e, vol. 4, no. 3, pp. 1-6, 2006.

[17] A. W. Moore, J. Schneider, and K. Deng, "Efficient locally weighted polynomial regression predictions," in Proceedings of the International Machine Learning Conference. San Mateo, CA: Morgan Kaufmann, 1997.

[18] S. K. Nayar, G. Krishnan, M. D. Grossberg, and R. Raskar, "Fast separation of direct and global components of a scene using high frequency illumination," ACM Trans. Graph., vol. 25, no. 3, pp. 935-944, 2006.

[19] J. P. Norton, Introduction to Identification.. $\quad$ London: Academic, 1986.

[20] D. T. V. Pawluk and R. D. Howe, "Dynamic lumped element response of the human fingerpad," ASME J. Biomech. Eng., vol. 121, pp. 605-611, 1999.

[21] D. T. V. Pawluk and R. D. Howe, "Dynamic contact mechanics of the human fingerpad against a flat surface," ASME J. Biomech. Eng., vol. 121, pp. 178-183, 1999 .

[22] C. L. Phillips and R. D. Harbor, Feedback Control Systems, 4th ed. Upper Saddle River, NJ: Prentic-Hall, 2000.

[23] G. A. F. Seber and A. J. Lee, Linear Regression Analysis.. Hoboken, NJ: Wiley, 2003.

[24] J. K. Shim, M. L. Latash, and V. M. Zatsiorsky, "Prehension synergies: Trial-to-trial variability and hierarchical organization of stable performance," Exp. Brain Res., vol. 152, pp. 173-184, 2003.

[25] J. K. Shim, M. L. Latash, and V. M. Zatsiorsky, "Prehension synergies in three dimentions," J. Newrophysiol., vol. 93, pp. 766-776, 2005.

[26] M. P. Rearick, A. Casares, and M. Santello, "Task-dependent modulation of multi-digit force coordination patterns," J. Neurophysiol., vol. 89, pp. 1317-1326, 2003.

[27] R. Reilmann, A. M. Gordon, and H. Henningsen, "Initiation and development of fingertip forces during whole-hand grasping," Exp. Brain Res., vol. 140, pp. 443-452, 2001.

[28] M. Santello and J. F. Soechting, "Force synergies for multifingered grasping," Exp. Brain Res., vol. 133, pp. 457-467, 2000.

[29] L. G. Shapiro and G. C. Stockman, Computer Vision. Upper Saddle River, NJ: Prentice-Hall, 2001. 
[30] Y. Sun, J. M. Hollerbach, and S. A. Mascaro, "Measuring fingertip forces by imaging the fingernail," in Proc. 14th Symp. Haptic Interfaces Virtual Environ. Teleoperator Syst., 2006, pp. 125-131.

[31] Y. Sun, J. M. Hollerbach, and S. A. Mascaro, "Dynamic features and prediction model for imaging the fingernail to measure fingertip forces," in Proc. IEEE Int. Conf. Robot. Autom., May 15-19, 2006, pp. 2813-2818.

[32] Y. Sun, J. M. Hollerbach, and S. A. Mascaro, "Imaging the finger force direction," in Proc. IEEE Comput. Vis. Pattern Recogn., Minneapolis, MN, Jun. 17-22, 2007, pp. 1-6.

[33] H. W. Trucco and V. Alessandro, Introductory Techniques for 3-D Computer Vision. Englewood Cliffs, NJ: Prentice-Hall, 1998.

[34] P. F. Verhulst, "A note on population growth," Corresp. Math. Phys., vol. 10, pp. 113-121, 1938.

[35] G. Westling and R. S. Johansson, "Factors influencing the force control during precision grip," Exp. Brain Res., vol. 53, pp. 277-284, 1984.

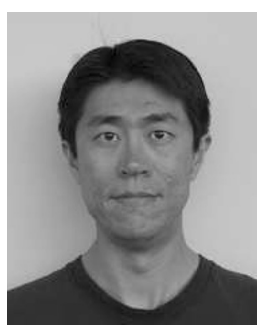

Yu Sun (S'03-M'07) received the B.S. and M.S. degrees in electrical engineering from Dalian University of Technology, Dalian, China, in 1997 and 2000, respectively, and the Ph.D. degree in computer science from the University of Utah, Salt Lake City, in 2007.

$\mathrm{He}$ is currently a Postdoctoral Associate in Mitsubishi Electric Research Laboratories, Cambridge, MA. His current research interests include robotics, computer vision, and haptics.

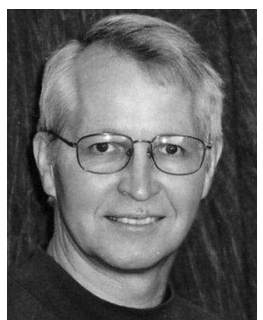

John M. Hollerbach (M'85-SM'92-F'96) received the B.S. degree in chemistry and the M.S. degree in mathematics from the University of Michigan, Ann Arbor, in 1968 and 1969, respectively, and the S.M. and Ph.D. degrees in computer science from Massachusetts Institute of Technology (MIT), Cambridge, in 1975 and 1978, respectively.

From 1978 to 1982, he was a Research Scientist. From 1982 to 1989 , he was a faculty member in the Department of Brain and Cognitive Sciences and a member of the Artificial Intelligence Laboratory, MIT. From 1989 to 1994, he was the Natural Sciences and Engineering/Canadian Institute for Advanced Research Professor of Robotics at McGill University, jointly in the Departments of Mechanical Engineering and Biomedical Engineering. He is currently a Professor in the School of Computing, University of Utah, Salt Lake City, where he directs the robotics track. He is also a Research Professor in mechanical engineering. His current research interests include combine robotics, virtual reality, and human motor control.

Prof. Hollerbach was the recipient of the 1984 National Science Foundation (NSF) Presidential Young Investigator Award. In 1988, he was named a Fellow of the Canadian Institute for Advanced Research. He was the Program Chairman of the 1989 IEEE International Conference on Robotics and Automation, a member of the Administrative Committee of the IEEE Robotics and Automation Society from 1989 to 1993, a Technical Editor of the IEEE TRANSACTIONS ON ROBOTICS AND AUTOMATION from 1989 to 1994, and a Treasurer of the IEEE/ASME JOURNAL OF MICROELECTROMECHANICAL SYSTEMS from 1992 to 1997. He was a member of the 1994-1995 National Research Council Committee on Virtual Reality Research and Development. He is currently an Editor of the International Journal of Robotics Research, and the Vice President for Conference Activities of the IEEE Robotics and Automation Society.

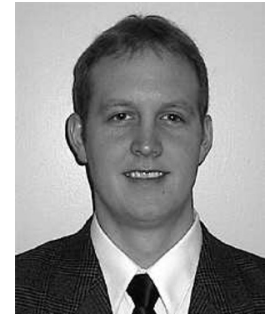

Stephen A. Mascaro (S'96-M'02) received the B.A. degree in physics from Houghton College, Houghton, $\mathrm{NY}$, and the B.S. degree in mechanical engineering from Clarkson University, Potsdam, NY, both in 1995, and the M.S. and Ph.D. degrees in mechanical engineering from Massachusetts Institute of Technology (MIT), Cambridge, in 1997 and 2002, respectively.

From 2002 to 2003, he was a Postdoctoral Associate in the Department of Mechanical Engineering, MIT, and an Assistant Professor of Mechanical Engineering at North Dakota State University from 2003 to 2004. Since 2005, he has been an Assistant Professor in the Department of Mechanical Engineering, University of Utah, Salt Lake City. His current research interests include robotics and control, system dynamics, human-machine interaction, haptics, and bioinspired actuation.

Dr. Mascaro was the recipient of the Best Paper Award at the 1999 IEEE International Conference on Robotics and Automation for his original work on fingernail sensors. He is also a member of the American Society of Mechanical Engineers. His original work on fingernail sensing was published in the IEEE TRANSACTIONS ON ROBOTICS AND AUTOMATION in 2001 and 2004. His original work on fingernail sensing was published in Haptics-e: The Electronic Journal of Haptics Research in 2006. 